\title{
FRACTIONAL LOGARITHMIC INEQUALITIES AND BLOW-UP RESULTS WITH LOGARITHMIC NONLINEARITY ON HOMOGENEOUS GROUPS
}

\author{
AIDYN KASSYMOV, MICHAEL RUZHANSKY, AND DURVUDKHAN SURAGAN
}

\begin{abstract}
In this paper, we prove the fractional logarithmic Sobolev inequality on homogeneous groups. Also, we establish fractional logarithmic Gagliardo-Nirenberg and Caffarelli-Kohn-Nirenberg inequalities on homogeneous groups. In addition, we show blow-up results for the fractional heat equation with logarithmic nonlinearity on homogeneous groups.
\end{abstract}

\section{Contents}



\section{INTRODUCTION}

First of all, let us briefly give an introduction to the considered results. The obtained inequalities seem new even in the Euclidean setting.

1.1. Fractional Sobolev inequality. Let $\Omega \subset \mathbb{R}^{N}$ be a measurable set and $1<$ $p<N$, then the (classical) Sobolev inequality is formulated as

$$
\|u\|_{L^{p^{*}}(\Omega)} \leq C\|\nabla u\|_{L^{p}(\Omega)}, \quad u \in C_{0}^{\infty}(\Omega),
$$

1991 Mathematics Subject Classification. 22E30, 43A80.

Key words and phrases. fractional logarithmic Sobolev inequality, fractional logarithmic Gagliardo-Nirenberg inequality, fractional logarithmic Caffarelli-Kohn-Nirenberg inequality, blowup, homogeneous group.

The authors were supported in parts by the FWO Odysseus Project, by the EPSRC grant EP/R003025/1 and by the Leverhulme Grant RPG-2017-151, as well as by the MESRK grant AP05130981. 
where $C=C(N, p)>0$ is a positive constant, $p^{*}=\frac{N p}{N-p}$ and $\nabla$ is the standard gradient in $\mathbb{R}^{N}$. The Sobolev inequality is one of the most important tools in PDE and variational problems.

In [6] the authors obtained the fractional Sobolev inequality in the case $N>s p$, $1<p<\infty$, and $s \in(0,1)$ :

$$
\|u\|_{L^{p^{*}}\left(\mathbb{R}^{N}\right)} \leq C[u]_{s, p},
$$

for any measurable and compactly supported function $u$ where $C=C(N, p, s)>0$ is a suitable constant,

$$
[u]_{s, p}^{p}=\int_{\mathbb{R}^{N}} \int_{\mathbb{R}^{N}} \frac{|u(x)-u(y)|^{p}}{|x-y|^{N+s p}} d x d y
$$

is the Gagliardo seminorm and $p^{*}=\frac{N p}{N-s p}$. There is a number of generalisations and extensions of the above Sobolev's inequality. For example, in [1] the authors proved the following weighted fractional Sobolev inequality: Let $1<p<\frac{N}{s}$ and $0<\beta<\frac{N-p s}{2}$, then for all $u \in C_{0}^{\infty}\left(\mathbb{R}^{N}\right)$ one has

$$
\left(\int_{\mathbb{R}^{N}} \frac{|u|^{p^{*}}}{|x|^{\frac{2 \beta p^{*}}{p}}} d x\right)^{\frac{p}{p^{*}}} \leq C \int_{\mathbb{R}^{N}} \int_{\mathbb{R}^{N}} \frac{|u(x)-u(y)|^{p}}{|x-y|^{N+p s}|x|^{\beta}|y|^{\beta}} d x d y
$$

where $C=C(N, p, s)>0$ and $p^{*}=\frac{N p}{N-s p}$.

In the homogeneous group setting, the fractional Sobolev inequality was obtained in [12]. In [15], the author proved the logarithmic Sobolev inequality in the following form:

$$
\int_{\mathbb{R}^{N}} \frac{|u|^{p}}{\|u\|_{L^{p}\left(\mathbb{R}^{N}\right)}^{p}} \log \left(\frac{|u|^{p}}{\|u\|_{L^{p}\left(\mathbb{R}^{N}\right)}^{p}}\right) d x \leq \frac{N}{p} \log \left(C \frac{\|\nabla u\|_{L^{p}\left(\mathbb{R}^{N}\right)}^{p}}{\|u\|_{L^{p}\left(\mathbb{R}^{N}\right)}^{p}}\right), 1 \leq p<\infty,
$$

where $u, \nabla u \in L^{p}\left(\mathbb{R}^{N}\right)$.

Combining the above ideas in [9], the authors proved a version of the fractional logarithmic Sobolev inequality. In this paper one of our aims is to obtain an analogue of the fractional logarithmic Sobolev inequality on the homogeneous groups.

We refer to [7] for the original appearance of such groups, and to [24] for a recent comprehensive treatment. However, for convinience, we shortly review basic facts needed in this paper in Section 2 .

1.2. Fractional Gagliardo-Nirenberg inequality. In the works of E. Gagliardo [8] and L. Nirenberg [16] (independently), they obtained the following (interpolation) inequality

$$
\|u\|_{L^{p}\left(\mathbb{R}^{N}\right)}^{p} \leq C\|\nabla u\|_{L^{2}\left(\mathbb{R}^{N}\right)}^{N(p-2) / 2}\|u\|_{L^{2}\left(\mathbb{R}^{N}\right)}^{(2 p-N(p-2)) / 2}, u \in H^{1}\left(\mathbb{R}^{N}\right),
$$

where

$$
\left\{\begin{array}{l}
2 \leq p \leq \infty \text { for } N=2 \\
2 \leq p \leq \frac{2 N}{N-2} \text { for } N>2
\end{array}\right.
$$

The Gagliardo-Nirenberg inequality on the Heisenberg group $\mathbb{H}^{n}$ has the following form

$$
\|u\|_{L^{p}\left(\mathbb{H}^{n}\right)}^{p} \leq C\left\|\nabla_{\mathbb{H}^{n}} u\right\|_{L^{2}\left(\mathbb{H}^{n}\right)}^{Q(p-2) / 2}\|u\|_{L^{2}\left(\mathbb{H}^{n}\right)}^{(2 p-Q(p-2)) / 2}
$$


where $\nabla_{\mathbb{H}}$ is a horizontal gradient and $Q=2 n+2$ is a homogeneous dimension of $\mathbb{H}^{n}$. In [5], the authors determined the best constant for the sub-elliptic GagliardoNirenberg inequality (1.6). Consequently, in [20] the best constants in GagliardoNirenberg and Sobolev inequalities were also found for general hypoelliptic operators (Rockland operators) on graded groups.

In [17] the authors obtained a fractional version of the Gagliardo-Nirenberg inequality in the form:

$$
\|u\|_{L^{\tau}\left(\mathbb{R}^{N}\right)} \leq C[u]_{s, p}^{a}\|u\|_{L^{\alpha}\left(\mathbb{R}^{N}\right)}^{1-a}, \forall u \in C_{c}^{1}\left(\mathbb{R}^{N}\right),
$$

for $N \geq 1, s \in(0,1), p>1, \alpha \geq 1, \tau>0$, and $a \in(0,1]$ is such that

$$
\frac{1}{\tau}=a\left(\frac{1}{p}-\frac{s}{N}\right)+\frac{1-a}{\alpha}
$$

Then this inequality was extended to homogeneous groups in [14. Also, the logarithmic Gagliardo-Nirenberg inequality with $s=1$ was proved in [15] and its fractional version was proved in [9]. In this paper we obtain the fractional logarithmic Gagliardo-Nirenberg inequality on the homogeneous groups.

Note that, to the best of our knowledge, in this direction systematic studies on the homogeneous groups started by the paper [18] in which homogeneous group versions of Hardy and Rellich inequalities were proved as consequences of universal identities resulting the recent book [24. Also, in [13] Hardy-Littlewood-Sobolev and SteinWeiss inequalities were obtained on homogeneous Lie groups.

1.3. Fractional Caffarelli-Kohn-Nirenberg inequality. In their pioneering work [2], L. Caffarelli, R. Kohn and L. Nirenberg established:

Theorem 1.1. Let $N \geq 1$, and let $l_{1}, l_{2}, l_{3}, a, b, d, \delta \in \mathbb{R}$ be such that $l_{1}, l_{2} \geq 1$, $l_{3}>0,0 \leq \delta \leq 1$, and

$$
\frac{1}{l_{1}}+\frac{a}{N}, \frac{1}{l_{2}}+\frac{b}{N}, \frac{1}{l_{3}}+\frac{\delta d+(1-\delta) b}{N}>0
$$

Then,

$$
\left\||x|^{\delta d+(1-\delta) b} u\right\|_{L^{l_{3}\left(\mathbb{R}^{N}\right)}} \leq C\left\||x|^{a} \nabla u\right\|_{L^{l_{1}\left(\mathbb{R}^{N}\right)}}^{\delta}\left\||x|^{b} u\right\|_{L^{l_{2}\left(\mathbb{R}^{N}\right)}}^{1-\delta}, \quad u \in C_{c}^{\infty}\left(\mathbb{R}^{N}\right),
$$

if and only if

$$
\begin{aligned}
\frac{1}{l_{3}}+\frac{\delta d+(1-\delta) b}{N}=\delta\left(\frac{1}{l_{1}}+\frac{a-1}{N}\right)+(1-\delta)\left(\frac{1}{l_{2}}+\frac{b}{N}\right), \\
a-d \geq 0, \quad \text { if } \delta>0 \\
a-d \leq 1, \quad \text { if } \delta>0 \text { and } \frac{1}{l_{3}}+\frac{\delta d+(1-\delta) b}{N}=\frac{1}{l_{1}}+\frac{a-1}{N},
\end{aligned}
$$

where $C$ is a positive constant independent of $u$.

In [17, the authors proved the fractional analogues of the Caffarelli-Kohn-Nirenberg inequality in the weighted fractional Sobolev spaces. The fractional Caffarelli-KohnNirenberg inequality for an admissible weight in $\mathbb{R}^{N}$ was obtained in [1]. The logarithmic analogue of the Caffarelli-Kohn-Nirenberg inequality was proved in [25]. 
Recently many different versions of Caffarelli-Kohn-Nirenberg inequalities have been obtained on different Lie groups, namely, in [26] on the Heisenberg groups, in [22] and [23] on stratified groups, in [19] and [21] on (general) homogeneous groups. On the homogeneous groups a fractional analogue of Caffarelli-Kohn-Nirenberg inequality was proved in [14]. One of the aims of this paper is to obtain the fractional logarithmic Caffarelli-Kohn-Nirenberg inequality on homogeneous groups.

1.4. Blow-up result for the fractional heat equation with logarithmic nonlinearity. In [3] the authors study the following initial boundary value problem:

$$
\left\{\begin{array}{l}
u_{t}(x, t)-\Delta_{x} u(x, t)=u \log |u|, \quad(x, t) \in \Omega \times(0,+\infty), \\
u(x, 0)=u_{0}(x), \quad x \in \Omega \\
u(x, t)=0, \quad(x, t) \in \partial \Omega \times(0,+\infty) .
\end{array}\right.
$$

So, they prove the following blow-up theorem (in the Euclidean setting):

Theorem 1.2. [3] Assume that $u_{0} \in H_{0}^{1}(\Omega)$ and

$$
J\left(u_{0}\right)=\frac{1}{2} \int_{\Omega}\left|\nabla u_{0}\right|^{2} d x-\frac{1}{2} \int_{\Omega}\left|u_{0}\right|^{2} \log \left|u_{0}\right| d x+\frac{1}{4} \int_{\Omega}\left|u_{0}\right|^{2} d x \leq M,
$$

and

$$
I\left(u_{0}\right)=\int_{\Omega}\left|\nabla u_{0}\right|^{2} d x-\int_{\Omega}\left|u_{0}\right|^{2} \log \left|u_{0}\right| d x<0 .
$$

Then the weak solution of the problem (1.11) blows up at $+\infty$.

Moreover, in [10] it is showed the condition $J\left(u_{0}\right) \leq M$ is unnecessary to blowup at infinity to a solution of the problem (1.11). We also refer to [4] and [1] to blow-up results for the pseudo-parabolic equations with logarithmic nonlinearity. In this paper, we considered the heat equation with the fractional sub-Laplacian with logarithmic nonlinearity and we obtain the blow-up result. That is, we extend the blow-up theorem from [10] to general homogeneous groups.

Summarising our main results of the present paper, we prove the following facts:

- An analogue of the fractional logarithmic Sobolev inequality on the homogeneous group $\mathbb{G}$;

- An analogue of the fractional logarithmic Gagliardo-Nirenberg inequality on $\mathbb{G}$;

- An analogue of the fractional logarithmic Caffarelli-Kohn-Nirenberg inequality on $\mathbb{G}$;

- Blow-up theorem for the fractional heat equation with the logarithmic nonlinearity on $\mathbb{G}$.

The paper is organised as follows. First we give some basic discussions on fractional Sobolev spaces and related facts on homogeneous groups, then in Section 3 we present the fractional logarithmic Sobolev, Gagliardo-Nirenberg, and CaffarelliKohn-Nirenberg inequalities on $\mathbb{G}$. In Section 4 we prove the blow-up theorem to the fractional heat equation with logarithmic nonlinearity on the homogeneous group $\mathbb{G}$. 


\section{Preliminaries}

We recall that a Lie group (on $\mathbb{R}^{n}$ ) $\mathbb{G}$ with the dilation

$$
D_{\lambda}(x):=\left(\lambda^{\nu_{1}} x_{1}, \ldots, \lambda^{\nu_{n}} x_{n}\right), \nu_{1}, \ldots, \nu_{n}>0, D_{\lambda}: \mathbb{R}^{n} \rightarrow \mathbb{R}^{n},
$$

which is an automorphism of the group $\mathbb{G}$ for each $\lambda>0$, is called a homogeneous (Lie) group. In this paper, for simplicity, we use the notation $\lambda x$ instead of the dilation $D_{\lambda}(x)$. The homogeneous dimension of the homogeneous group $\mathbb{G}$ is denoted by

$$
Q:=\nu_{1}+\ldots+\nu_{n}
$$

A homogeneous quasi-norm on $\mathbb{G}$ is a continuous non-negative function

$$
\mathbb{G} \ni x \mapsto|x| \in[0, \infty),
$$

with the properties

i) $|x|=\left|x^{-1}\right|$ for all $x \in \mathbb{G}$,

ii) $|\lambda x|=\lambda|x|$ for all $x \in \mathbb{G}$ and $\lambda>0$,

iii) $|x|=0$ iff $x=0$.

Moreover, the following polarisation formula on homogeneous groups will be used in our proofs: there is a (unique) positive Borel measure $\sigma$ on the unit quasi-sphere $\mathfrak{S}:=\{x \in \mathbb{G}:|x|=1\}$, so that for every $f \in L^{1}(\mathbb{G})$ we have

$$
\int_{\mathbb{G}} f(x) d x=\int_{0}^{\infty} \int_{\mathfrak{S}} f(r y) r^{Q-1} d \sigma(y) d r .
$$

We refer to [7] and a recent open access book [24] for more details.

Let us define quasi-ball centered at $x$ with radius $r$ in the following form:

$$
B(x, r):=\left\{x \in \mathbb{G}:\left|x^{-1} y\right|<r\right\} .
$$

Let $s \in(0,1)$ and let $\mathbb{G}$ be a homogeneous group of homogeneous dimension $Q$. For a (Haar) measurable and compactly supported function $u$ the fractional sub-Laplacian $\left(-\Delta_{s}\right)$ on $\mathbb{G}$ can be defined as

$$
\left(-\Delta_{s}\right) u(x):=2 \lim _{r \searrow 0} \int_{\mathbb{G} \backslash B(x, r)} \frac{u(x)-u(y)}{\left|y^{-1} x\right|^{Q+2 s}} d y, \quad x \in \mathbb{G},
$$

where $|\cdot|$ is a quasi-norm on $\mathbb{G}$ and $B(x, r)$ is a quasi-ball with respect to $|\cdot|$, with radius $r$ centered at $x \in \mathbb{G}$.

Let $p>1$. For a measurable function $u: \mathbb{G} \rightarrow \mathbb{R}$ we define the Gagliardo quasiseminorm by

$$
[u]_{s, p}:=\left(\int_{\mathbb{G}} \int_{\mathbb{G}} \frac{|u(x)-u(y)|^{p}}{\left|y^{-1} x\right|^{Q+s p}} d x d y\right)^{1 / p} .
$$

Now we recall the definition of the fractional Sobolev spaces on homogeneous groups denoted by $W^{s, p}(\mathbb{G})$. For $p \geq 1$ and $s \in(0,1)$, the functional space

$$
W^{s, p}(\mathbb{G})=\left\{u \in L^{p}(\mathbb{G}): u \text { is measurable, }[u]_{s, p}<+\infty\right\},
$$

is called the fractional Sobolev space on $\mathbb{G}$. 
Similarly, if $\Omega \subset \mathbb{G}$ is a Haar measurable set, we define the Sobolev space $W^{s, p}(\Omega)=\left\{u \in L^{p}(\Omega): u\right.$ is measurable,

$$
\left.[u]_{s, p, \Omega}=\left(\int_{\Omega} \int_{\Omega} \frac{|u(x)-u(y)|^{p}}{\left|y^{-1} x\right|^{Q+s p}} d x d y\right)^{\frac{1}{p}}<+\infty\right\} .
$$

Let us define $W_{0}^{s, p}(\Omega)$ as the completion of $C_{0}^{\infty}(\Omega)$ with respect to the norm

$$
\|u\|_{W_{0}^{s, p}(\Omega)}=[u]_{s, p, \Omega} .
$$

In the case $p=2$, for all $\varphi \in W_{0}^{s, 2}(\Omega)$ we have

$$
\left\langle\left(-\Delta_{s}\right) u, \varphi\right\rangle:=\int_{\Omega} \int_{\Omega} \frac{(u(x)-u(y))(\varphi(x)-\varphi(y))}{\left|y^{-1} x\right|^{Q+2 s}} d x d y .
$$

Now we recall the definition of the weighted fractional Sobolev space on the homogeneous groups denoted by

$$
\begin{aligned}
& W^{s, p, \beta}(\mathbb{G})=\left\{u \in L^{p}(\mathbb{G}): u\right. \text { is measurable, } \\
& \left.\qquad u]_{s, p, \beta}=\left(\int_{\mathbb{G}} \int_{\mathbb{G}} \frac{|x|^{\beta_{1} p}|y|^{\beta_{2} p}|u(x)-u(y)|^{p}}{\left|y^{-1} x\right|^{Q+s p}} d x d y\right)^{\frac{1}{p}}<+\infty\right\},
\end{aligned}
$$

where $\beta_{1}, \beta_{2} \in \mathbb{R}$ with $\beta=\beta_{1}+\beta_{2}$, that is, it depends on $\beta_{1}$ and $\beta_{2}$.

As above, for a Haar measurable set $\Omega \subset \mathbb{G}, p \geq 1, s \in(0,1)$ and $\beta_{1}, \beta_{2} \in \mathbb{R}$ with $\beta=\beta_{1}+\beta_{2}$, we define the weighted fractional Sobolev space

$$
W^{s, p, \beta}(\Omega)=\left\{u \in L^{p}(\Omega): u\right. \text { is measurable, }
$$

$$
\left.[u]_{s, p, \beta, \Omega}=\left(\int_{\Omega} \int_{\Omega} \frac{|x|^{\beta_{1} p}|y|^{\beta_{2} p}|u(x)-u(y)|^{p}}{\left|y^{-1} x\right|^{Q+s p}} d x d y\right)^{\frac{1}{p}}<+\infty\right\} .
$$

Obviously, taking $\beta=\beta_{1}=\beta_{2}=0$ in (2.11), we recover (2.7).

\section{Fractional Logarithmic inequalities on $\mathbb{G}$}

In this section we prove analogues of fractional logarithmic inequalities on the homogeneous groups. From now on, unless specified otherwise, $\mathbb{G}$ will be a homogeneous group of homogeneous dimension $Q$. Firstly, we show weighted Hölder's inequality on $\mathbb{G}$.

Lemma 3.1. Suppose that $1<p \leq r \leq q \leq \infty, a \in[0,1], \alpha \in \mathbb{R},|x|^{\alpha} u \in$ $L^{p}(\mathbb{G}) \cap L^{q}(\mathbb{G})$ with

$$
\frac{1}{r}=\frac{a}{p}+\frac{1-a}{q}
$$

then we have

$$
\left\||x|^{\alpha} u\right\|_{L^{r}(\mathbb{G})} \leq\left\||x|^{\alpha} u\right\|_{L^{p}(\mathbb{G})}^{a}\left\||x|^{\alpha} u\right\|_{L^{q}(\mathbb{G})}^{1-a} .
$$


Proof. By using Hölder's inequality we obtain

$$
\begin{aligned}
\left\||x|^{\alpha} u\right\|_{L^{r}(\mathbb{G})}^{r} & =\int_{\mathbb{G}}|x|^{\alpha r}|u(x)|^{r} d x=\int_{\mathbb{G}}\left(|x|^{\alpha}|u(x)|\right)^{a r}\left(|x|^{\alpha}|u(x)|\right)^{(1-a) r} d x \\
& \leq\left(\int_{\mathbb{G}}|x|^{\alpha p}|u(x)|^{p} d x\right)^{\frac{a r}{p}}\left(\int_{\mathbb{G}}|x|^{\alpha q}|u(x)|^{q} d x\right)^{\frac{(1-a) r}{q}} \\
& =\left\||x|^{\alpha} u\right\|_{L^{p}(\mathbb{G})}^{a r}\left|\left\|\left.x\right|^{\alpha} u\right\|_{L^{q}(\mathbb{G})}^{(1-a) r},\right.
\end{aligned}
$$

with

$$
\frac{a r}{p}+\frac{(1-a) r}{q}=1
$$

Now let us present logarithmic Hölder's inequality.

Lemma 3.2. Let $|x|^{\alpha} u \in L^{p}(\mathbb{G}) \cap L^{q}(\mathbb{G})$ with some $\alpha \in \mathbb{R}, 1<p<q \leq \infty$. Then we have

$$
\int_{\mathbb{G}} \frac{\left(|x|^{\alpha p}|u|^{p}\right)}{\left\||x|^{\alpha} u\right\|_{L^{p}(\mathbb{G})}^{p}} \log \left(\frac{|x|^{\alpha p}|u|^{p}}{\left\||x|^{\alpha} u\right\|_{L^{p}(\mathbb{G})}^{p}}\right) d x \leq \frac{q}{q-p} \log \left(\frac{\left\||x|^{\alpha} u\right\|_{L^{q}(\mathbb{G})}^{p}}{\left\||x|^{\alpha} u\right\|_{L^{p}(\mathbb{G})}^{p}}\right) .
$$

Proof. Consider the function

$$
F\left(\frac{1}{r}\right)=\log \left(\left\||x|^{\alpha} u\right\|_{L^{r}(\mathbb{G})}\right) .
$$

Now let us show that the function (3.6) is convex. To prove it we use Lemma 3.1, so we get

$$
\begin{aligned}
& F\left(\frac{1}{r}\right)=\log \left(\left\||x|^{\alpha} u\right\|_{L^{r}(\mathbb{G})}\right) \leq \log \left(\left\||x|^{\alpha} u\right\|_{L^{p}(\mathbb{G})}^{a}\left\||x|^{\alpha} u\right\|_{L^{q}(\mathbb{G})}^{1-a}\right) \\
& =\log \left(\left\||x|^{\alpha} u\right\|_{L^{p}(\mathbb{G})}^{a}\right)+\log \left(\left\||x|^{\alpha} u\right\|_{L^{q}(\mathbb{G})}^{1-a}\right)=a F\left(\frac{1}{p}\right)+(1-a) F\left(\frac{1}{q}\right)
\end{aligned}
$$

with $a \in[0,1]$ and $\frac{1}{r}=\frac{a}{p}+\frac{1-a}{q}$.

Since we have

$$
F(r)=r \log \int_{\mathbb{G}}|x|^{\frac{\alpha}{r}}|u(x)|^{\frac{1}{r}} d x
$$

the derivative of $(3.8)$ is

$$
\begin{aligned}
F^{\prime}(r)=\log \int_{\mathbb{G}}|x|^{\frac{\alpha}{r}}|u(x)|^{\frac{1}{r}} d x+r\left(\log \int_{\mathbb{G}}|x|^{\frac{\alpha}{r}}|u(x)|^{\frac{1}{r}} d x\right)_{r}^{\prime} \\
\quad=\log \int_{\mathbb{G}}|x|^{\frac{\alpha}{r}}|u(x)|^{\frac{1}{r}} d x+r \frac{\left(\int_{\mathbb{G}}\left(|x|^{\alpha} u(x)\right)^{\frac{1}{r}} d x\right)_{r}^{\prime}}{\int_{\mathbb{G}}|x|^{\frac{\alpha}{r}}|u(x)|^{\frac{1}{r}} d x} \\
=\log \int_{\mathbb{G}}|x|^{\frac{\alpha}{r}}|u(x)|^{\frac{1}{r}} d x-\frac{1}{r} \frac{\int_{\mathbb{G}}\left(|x|^{\alpha} u(x)\right)^{\frac{1}{r}} \log \left(|x|^{\alpha}|u(x)|\right) d x}{\int_{\mathbb{G}}|x|^{\frac{\alpha}{r}}|u(x)|^{\frac{1}{r}} d x} .
\end{aligned}
$$


On the other hand, by (3.7) $F(r)$ is convex, therefore, we have

$$
F^{\prime}(r) \geq \frac{F\left(r^{\prime}\right)-F(r)}{r^{\prime}-r}, \quad r^{\prime}>r>0 .
$$

With $r=\frac{1}{p}$ and $r^{\prime}=\frac{1}{q}$ it yields

$$
p \frac{\left.\left.\int_{\mathbb{G}}|| x\right|^{\alpha} u\right|^{p} \log |x|^{\alpha}|u| d x}{\int_{\mathbb{G}}|x|^{\alpha p}|u(x)|^{p} d x}-\log \int_{\mathbb{G}}|x|^{\alpha p}|u(x)|^{p} d x \leq \frac{q p}{q-p} \log \left(\int_{\mathbb{G}} \frac{\left\||x|^{\alpha} u\right\|_{L^{q}(\mathbb{G})}}{\left\||x|^{\alpha} u\right\|_{L^{p}(\mathbb{G})}}\right) .
$$

We have

$$
\begin{array}{r}
\log \int_{\mathbb{G}}|x|^{\alpha p}|u(x)|^{p} d x=\frac{\log \int_{\mathbb{G}}|x|^{\alpha p}|u(x)|^{p} d x \int_{\mathbb{G}}|x|^{\alpha p}|u(x)|^{p} d x}{\int_{\mathbb{G}}|x|^{\alpha p}|u(x)|^{p} d x} \\
=\frac{\int_{\mathbb{G}}|x|^{\alpha p}|u(x)|^{p} \log \left\||x|^{\alpha} u\right\|_{L^{p}(\mathbb{G})}^{p} d x}{\int_{\mathbb{G}}|x|^{\alpha p}|u(x)|^{p} d x} .
\end{array}
$$

Substituting it in (3.11) we obtain logarithmic Hölder's inequality

$$
\begin{aligned}
p \frac{\left.\left.\int_{\mathbb{G}}|| x\right|^{\alpha} u\right|^{p} \log |x|^{\alpha}|u| d x}{\int_{\mathbb{G}}|x|^{\alpha p}|u(x)|^{p} d x}-\log \int_{\mathbb{G}}|x|^{\alpha p}|u(x)|^{p} d x \\
=p \frac{\left.\left.\int_{\mathbb{G}}|| x\right|^{\alpha} u\right|^{p} \log |x|^{\alpha}|u| d x}{\int_{\mathbb{G}}|x|^{\alpha p}|u(x)|^{p} d x}-\frac{\int_{\mathbb{G}}|x|^{\alpha p}|u(x)|^{p} \log \left\||x|^{\alpha} u\right\|_{L^{p}(\mathbb{G})}^{p} d x}{\int_{\mathbb{G}}|x|^{\alpha p}|u(x)|^{p} d x} \\
=\frac{\left.\left.\int_{\mathbb{G}}|| x\right|^{\alpha} u\right|^{p} \log |x|^{\alpha p}|u|^{p} d x}{\int_{\mathbb{G}}|x|^{\alpha p}|u(x)|^{p} d x}-\frac{\int_{\mathbb{G}}|x|^{\alpha p}|u(x)|^{p} \log \left\||x|^{\alpha} u\right\|_{L^{p}(\mathbb{G})}^{p} d x}{\int_{\mathbb{G}}|x|^{\alpha p}|u(x)|^{p} d x} \\
=\int_{\mathbb{G}} \frac{\left(|x|^{\alpha p}|u|^{p}\right)}{\|\left.|x|^{\alpha}|u|\right|_{L^{p}(\mathbb{G})} ^{p}} \log \left(\frac{|x|^{\alpha p}|u|^{p}}{\left\||x|^{\alpha} u\right\|_{L^{p}(\mathbb{G})}^{p}}\right) \leq \frac{q}{q-p} \log \left(\frac{\left\|\left.x\right|^{\alpha} u\right\|_{L^{q}(\mathbb{G})}^{p}}{\left\||x|^{\alpha} u\right\|_{L^{p}(\mathbb{G})}^{p}}\right) .
\end{aligned}
$$

To prove fractional logarithmic Sobolev's inequality we need the version of a fractional Sobolev inequality on the homogeneous groups.

Theorem 3.3 ([12], Fractional Sobolev inequality). Let $\mathbb{G}$ be a homogeneous group with homogeneous dimension $Q$. Let $p>1, s \in(0,1), Q>s p$, and let $|\cdot|$ be a quasi-norm on $\mathbb{G}$. For any measurable and compactly supported function $u: \mathbb{G} \rightarrow \mathbb{R}$ there exists a positive constant $C=C(Q, p, s, q)>0$ such that

$$
\|u\|_{L^{p^{*}}(\mathbb{G})} \leq C[u]_{s, p}
$$

where $p^{*}=p^{*}(Q, s)=\frac{Q p}{Q-s p}$.

Now we present the fractional logarithmic Sobolev inequality on $\mathbb{G}$.

Theorem 3.4. Under the assumptions of Theorem 3.3 we have the fractional logarithmic Sobolev's inequality

$$
\int_{\mathbb{G}} \frac{|u|^{p}}{\|u\|_{L^{p}(\mathbb{G})}^{p}} \log \left(\frac{|u|^{p}}{\|u\|_{L^{p}(\mathbb{G})}^{p}}\right) d x \leq \frac{Q}{s p} \log \left(C \frac{[u]_{s, p}^{p}}{\|u\|_{L^{p}(\mathbb{G})}^{p}}\right),
$$


for any measurable and compactly supported function $u$. Here $C$ is a positive constant indefendent on $u$.

Proof. By using weighted logarithmic Hölder's inequality (3.5) with $\alpha=0$, we get

$$
\int_{\mathbb{G}} \frac{|u|^{p}}{\|u\|_{L^{p}(\mathbb{G})}^{p}} \log \left(\frac{|u|^{p}}{\|u\|_{L^{p}(\mathbb{G})}^{p}}\right) d x \leq \frac{q}{q-p} \log \left(\frac{\|u\|_{L^{q}(\mathbb{G})}^{p}}{\|u\|_{L^{p}(\mathbb{G})}^{p}}\right) .
$$

By the assumption we have $1 \leq p<q=p^{*}=\frac{p Q}{Q-s p}$, that is,

$$
\begin{aligned}
\int_{\mathbb{G}} \frac{|u|^{p}}{\|u\|_{L^{p}(\mathbb{G})}^{p}} \log \left(\frac{|u|^{p}}{\|u\|_{L^{p}(\mathbb{G})}^{p}}\right) d x \leq \frac{p^{*}}{p^{*}-p} \log \left(\frac{\|u\|_{L^{p^{*}(\mathbb{G})}}^{p}}{\|u\|_{L^{p}(\mathbb{G})}^{p}}\right) \\
\leq \frac{p^{*}}{p^{*}-p} \log \left(C \frac{[u]_{s, p}^{p}}{\|u\|_{L^{p}(\mathbb{G})}^{p}}\right) .
\end{aligned}
$$

Here we have

$$
\frac{p^{*}}{p^{*}-p}=\frac{\frac{p Q}{Q-s p}}{\frac{p Q}{Q-s p}-p}=\frac{\frac{Q}{Q-s p}}{\frac{Q}{Q-s p}-1}=\frac{Q}{s p} \text {. }
$$

Remark 3.5. In the Abelian (Euclidean) case $\mathbb{G}=\left(\mathbb{R}^{N},+\right)$, we have $Q=N$ and $|\cdot|=|\cdot|_{E}\left(|\cdot|_{E}\right.$ is the Euclidean distance), if $s \rightarrow 1^{-}$and from (3.15) we get the logarithmic Sobolev inequality from [15].

Let us recall the fractional logarithmic Gagliardo-Nirenberg inequality

Theorem 3.6 ([14], Fractional Gagliardo-Nirenberg inequality). Let $\mathbb{G}$ be a homogeneous group with homogeneous dimension $Q$. Assume that $Q \geq 2, s \in(0,1), p>1$, $q \geq 1, \tau>0, a \in(0,1], Q>s p$ and

$$
\frac{1}{\tau}=a\left(\frac{1}{p}-\frac{s}{Q}\right)+\frac{1-a}{q} .
$$

Then,

$$
\|u\|_{L^{\tau}(\mathbb{G})} \leq C[u]_{s, p}^{a}\|u\|_{L^{q}(\mathbb{G})}^{1-a}
$$

for all measurable and compactly supported $u$ and $C=C(s, p, Q, a, \alpha)>0$.

We have the following logarithmic fractional Gagliardo-Nirenberg inequality.

Theorem 3.7. Under the assumptions of Theorem 3.6 with the parameters $1 \leq p<$ $\infty, 1<q<\infty$ with $q \leq p^{*}$, there exists $C=C(Q, p, s, q)>0$ such that for all measurable and compactly supported $u$ we have

$$
\int_{\mathbb{G}} \frac{|u|^{q}}{\|u\|_{L^{q}(\mathbb{G})}^{q}} \log \left(\frac{|u|^{q}}{\|u\|_{L^{q}(\mathbb{G})}^{q}}\right) d x \leq \frac{1}{1-\frac{q}{\tau}} \log \left(C \frac{[u]_{s, p}^{q}}{\|u\|_{L^{q}(\mathbb{G})}^{q}}\right) d x .
$$


Proof. Combining the fractional Gagliardo-Nirenberg inequality (3.18) and the logarithmic Hölder inequality (3.5), we obtain

$$
\begin{aligned}
\int_{\mathbb{G}} \frac{|u|^{q}}{\|u\|_{L^{q}(\mathbb{G})}^{q}} \log \left(\frac{|u|^{q}}{\|u\|_{L^{q}(\mathbb{G})}^{q}}\right) d x \leq \frac{1}{1-\frac{q}{\tau}} \log \left(\frac{\|u\|_{L^{\tau}(\mathbb{G})}^{q}}{\|u\|_{q}^{q}}\right) \\
\leq \frac{1}{1-\frac{q}{\tau}} \log \left(C \frac{[u]_{s, p}^{q a}\|u\|_{L^{q}(\mathbb{G})}^{(1-a) q}}{\|u\|_{L^{q}(\mathbb{G})}^{q}}\right)=\frac{a}{1-\frac{q}{\tau}} \log \left(C \frac{[u]_{s, p}^{q}}{\|u\|_{L^{q}(\mathbb{G})}^{q}}\right) .
\end{aligned}
$$

Remark 3.8. In the Abelian (Euclidean) case $\mathbb{G}=\left(\mathbb{R}^{N},+\right)$, we have $Q=N$ and $|\cdot|=|\cdot|_{E}\left(|\cdot|_{E}\right.$ is the Euclidean distance), if $s \rightarrow 1^{-}$and from (3.19) we get the logarithmic Sobolev inequality in [15].

Recall the fractional Caffarelli-Kohn-Nirenberg (CKN) inequality on homogeneous groups.

Theorem 3.9 ([14], Fractional CKN inequality). Let $\mathbb{G}$ be a homogeneous group with homogeneous dimension $Q$. Assume that $Q \geq 2, s \in(0,1), p>1, q \geq 1, \tau>0$, $a \in(0,1], \beta_{1}, \beta_{2}, \beta, \mu, \gamma \in \mathbb{R}, \beta_{1}+\beta_{2}=\beta$ and

$$
\frac{1}{\tau}+\frac{\gamma}{Q}=a\left(\frac{1}{p}+\frac{\beta-s}{Q}\right)+(1-a)\left(\frac{1}{q}+\frac{\mu}{Q}\right) .
$$

Assume in addition that, $0 \leq \beta-\sigma$ with $\gamma=a \sigma+(1-a) \mu$, and

$$
\beta-\sigma \leq s \text { only if } \frac{1}{\tau}+\frac{\gamma}{Q}=\frac{1}{p}+\frac{\beta-s}{Q} .
$$

Then for all measurable and compactly supported $u$ we have

$$
\left\||x|^{\gamma} u\right\|_{L^{\tau}(\mathbb{G})} \leq C[u]_{s, p, \beta}^{a}\left\||x|^{\mu} u\right\|_{L^{q}(\mathbb{G})}^{1-a},
$$

when $\frac{1}{\tau}+\frac{\gamma}{Q}>0$, and for $u \in C_{c}^{1}(\mathbb{G} \backslash\{e\})$ we have

$$
\left\||x|^{\gamma} u\right\|_{L^{\tau}(\mathbb{G})} \leq C[u]_{s, p, \beta}^{a}\left\||x|^{\mu} u\right\|_{L^{q}(\mathbb{G})}^{1-a},
$$

when $\frac{1}{\tau}+\frac{\gamma}{Q}<0$. Here $e$ is the identity element of $\mathbb{G}$.

Now we present the fractional logarithmic CKN type inequality on homogeneous groups.

Theorem 3.10. Under the assumptions of Theorem 3.9 with

$$
\alpha=\beta=\mu, 1<q<p^{*}, 1<p<Q, \beta p+Q>0, \beta q+Q>0,
$$

there exists a positive constant $C$ such that

$$
\int_{\mathbb{G}} \frac{\left(|x|^{\alpha}|u|\right)^{q}}{\left\||x|^{\alpha} u\right\|_{L^{q}(\mathbb{G})}^{q}} \log \left(\frac{|x|^{\alpha q}|u|^{q}}{\left\||x|^{\alpha} u\right\|_{L^{q}(\mathbb{G})}^{q}}\right) d x \leq \frac{1}{1-\frac{q}{p^{*}}} \log \left(\frac{[u]_{s, p, \alpha}}{\left\||x|^{\alpha} u\right\|_{L^{q}(\mathbb{G})}^{q}}\right),
$$

for all measurable and compactly supported $u$. 
Proof. From the assumptions of Theorem 3.9 with $\alpha=\beta=\gamma$, we obtain that

$$
\frac{1}{\tau}=\frac{a}{p^{*}}+\frac{1-a}{q}
$$

From this identity with $q<p^{*}$ we get $q<\tau$. By using these facts with weighted logarithmic Hölder's inequality and $\alpha=\beta=\gamma$ we obtain

$$
\begin{aligned}
& \int_{\mathbb{G}} \frac{|x|^{\alpha q}|u|^{q}}{\left\||x|^{\alpha} u\right\|_{L^{q}(\mathbb{G})}^{q}} \log \left(\frac{|x|^{\alpha q}|u|^{q}}{\left\||x|^{\alpha} u\right\|_{L^{q}(\mathbb{G})}^{q}}\right) d x \leq \frac{\tau}{\tau-q} \log \left(\frac{\left\||x|^{\alpha} u\right\|_{L^{\tau}(\mathbb{G})}^{q}}{\left\||x|^{\alpha} u\right\|_{L^{q}(\mathbb{G})}^{q}}\right) \\
& \leq \frac{\tau}{\tau-q} \log \left(C^{a} \frac{[u]_{s, p, \alpha}^{a q}\left\||x|^{\alpha} u\right\|_{L^{q}(\mathbb{G})}^{(1-a) q}}{\left\||x|^{\alpha} u\right\|_{L^{q}(\mathbb{G})}^{q}}\right) \\
& =\frac{a \tau}{\tau-q} \log \left(C \frac{[u]_{s, p, \alpha}^{q}}{\left\||x|^{\alpha} u\right\|_{L^{q}(\mathbb{G})}^{q}}\right) \text {. }
\end{aligned}
$$

Since $\alpha=\beta=\gamma$, we have

$$
\frac{a \tau}{\tau-q}=\frac{p^{*}}{p^{*}-q}
$$

Remark 3.11. In the Abelian (Euclidean) case $\mathbb{G}=\left(\mathbb{R}^{N},+\right)$, we have $Q=N$ and $|\cdot|=|\cdot|_{E}\left(|\cdot|_{E}\right.$ is the Euclidean distance), if $s \rightarrow 1^{-}$and from $(3.26)$ we get the logarithmic Sobolev inequality in [25].

\section{BLOW-UP THEOREM ON $\mathbb{G}$}

Let us consider the following Cauchy-Dirichlet fractional heat equation on the homogeneous group:

$$
\left\{\begin{array}{l}
\frac{\partial u(x, t)}{\partial t}+\left(-\Delta_{s}\right) u(x, t)=u(x, t) \log |u(x, t)|, \quad(x, t) \in \Omega \times(0,+\infty), \Omega \subset \mathbb{G}, \\
u(x, t)=0, \quad(x, t) \in \mathbb{G} \backslash \Omega \times(0,+\infty), \\
u(x, 0)=u_{0}(x)
\end{array}\right.
$$

where $\Delta_{s}$ is the fractional sub-Laplacian with $s \in(0,1)$.

For simplicity, we introduce the notations $H_{0}^{s}(\Omega):=W_{0}^{s, 2}(\Omega)$ and $[u]_{s}:=[u]_{s, 2, \Omega}$. Let us recall the definition of a weak solution.

Definition 4.1. Let $T>0$. A function $u: \Omega \times[0,+\infty) \rightarrow \mathbb{R}, u=u(x, t) \in$ $L^{\infty}\left(0, T ; H_{0}^{s}(\Omega)\right)$ with $\frac{\partial u}{\partial t} \in L^{2}\left(0, T ; L^{2}(\Omega)\right)$ is a called a weak solution of problem (4.1) in $\Omega \times[0,+\infty)$, if $u_{0} \in H_{0}^{s}(\Omega)$ and $u$ satisfies (4.1) in the sense of distribution,

$$
\int_{\Omega} u_{t} \varphi d x+\left\langle\left(-\Delta_{s}\right) u, \varphi\right\rangle=\int_{\Omega} u \log |u| \varphi d x
$$

for any $\varphi \in H_{0}^{s}(\Omega), t \in(0,+\infty)$.

Definition 4.2. Let $u(x, t)$ be a weak solution of (4.1). We say that $u(x, t)$ blows up at $+\infty$ if

$$
\lim _{t \rightarrow+\infty}\|u(t, \cdot)\|_{L^{2}(\Omega)}^{2}=+\infty
$$


Let us consider the following energy functionals

$$
J(u)=\frac{1}{2}[u]_{s}^{2}-\frac{1}{2} \int_{\Omega} u^{2} \log |u| d x+\frac{1}{4} \int_{\Omega}|u|^{2} d x,
$$

and

$$
I(u)=[u]_{s}^{2}-\int_{\Omega} u^{2} \log |u| d x .
$$

Then we have

$$
J(u)=\frac{1}{2} I(u)+\frac{1}{4} \int_{\Omega}|u|^{2} d x .
$$

We have the following energy identity for (4.1).

Lemma 4.3. Suppose that $u$ is a weak solution of the problem (4.1). Then we have

$$
\int_{0}^{t}\left\|u_{\tau}\right\|_{L^{2}(\Omega)}^{2} d \tau+J(u)=J\left(u_{0}\right), \quad \forall t \in(0,+\infty) .
$$

Proof. Multiplying by $u_{t}$ and integrating over $\Omega$ in (4.1), we get

$$
\int_{\Omega}\left|u_{t}\right|^{2} d x+\left\langle\left(-\Delta_{s}\right) u, u_{t}\right\rangle=\int_{\Omega} u_{t} u \log |u| d x .
$$

For the second term on the left hand side of (4.8), we have

$$
\begin{aligned}
\left\langle\left(-\Delta_{s}\right) u, u_{t}\right\rangle=\int_{\Omega} \int_{\Omega} \frac{(u(x, t)-u(y, t))\left(u_{t}(x, t)-u_{t}(y, t)\right)}{\left|y^{-1} x\right|^{Q+2 s}} d x d y & =\frac{1}{2} \frac{d}{d t} \int_{\Omega} \int_{\Omega} \frac{|u(x, t)-u(y, t)|^{2}}{\left|y^{-1} x\right|^{Q+2 s}} d x d y=\frac{1}{2} \frac{d[u(t)]_{s}^{2}}{d t} .
\end{aligned}
$$

On the right hand side of (4.8), we have

$$
\frac{d u^{2} \log |u|}{d t}=2 u_{t} u \log |u|+u u_{t},
$$

then

$$
\begin{aligned}
\int_{\Omega} u_{t} u \log |u| d x=\frac{1}{2} \frac{d}{d t} \int_{\Omega} u^{2} \log |u| d x & -\frac{1}{2} \int_{\Omega} u u_{t} d x \\
& =\frac{1}{2} \frac{d}{d t} \int_{\Omega} u^{2} \log |u| d x-\frac{1}{4} \frac{d}{d t} \int_{\Omega} u^{2} d x .
\end{aligned}
$$

Plugging 4.9) and 4.11) in (4.8), we get

$$
\begin{aligned}
\int_{\Omega}\left|u_{t}\right|^{2} d x+\frac{d}{d t}\left(\frac{1}{2}[u]_{s}^{2}-\frac{1}{2} \int_{\Omega} u^{2} \log |u| d x+\frac{1}{4}\right. & \left.\int_{\Omega} u^{2} d x\right) \\
& =\int_{\Omega}\left|u_{t}\right|^{2} d x+\frac{d}{d t} J(u)=0 .
\end{aligned}
$$

Integrating over $(0, t)$, we arrive at

$$
\int_{0}^{t}\left\|u_{\tau}\right\|_{L^{2}(\Omega)}^{2} d \tau+\int_{0}^{t} \frac{d J(u)}{d \tau} d \tau=0
$$


that is,

$$
\int_{0}^{t}\left\|u_{\tau}\right\|_{L^{2}(\Omega)}^{2} d \tau+J(u)=J\left(u_{0}\right)
$$

Now we are in the position to present the main result of this section.

Theorem 4.4. Suppose that $u$ is a weak solution of (4.1) with $u_{0} \in H_{0}^{s}(\Omega)$ and $I\left(u_{0}\right)<0$. Then

$$
\lim _{t \rightarrow+\infty}\|u(t, \cdot)\|_{L^{2}(\Omega)}^{2}=+\infty .
$$

Proof. Firstly, by using (4.2) with $u=\varphi$ we get

$$
\begin{aligned}
\frac{d}{d t}\|u\|_{L^{2}(\Omega)}^{2}=\frac{d}{d t} \int_{\Omega} u^{2} d x & =2 \int_{\Omega} u u_{t} d x \\
& =-2\left(\int_{\Omega}\left\langle\left(-\Delta_{s}\right) u, u\right\rangle-u^{2} \log |u| d x\right)=-2 I(u) .
\end{aligned}
$$

So by using this, 4.2 and (4.6), we obtain

$$
\begin{gathered}
\frac{d I(u)}{d t}=\frac{d}{d t}\left(2 J(u)-\frac{1}{2} \int_{\Omega} u^{2} d x\right)=2 \int_{\Omega} \int_{\Omega} \frac{(u(x, t)-u(y, t))\left(u_{t}(x, t)-u_{t}(y, t)\right)}{\left|y^{-1} x\right|^{Q+2 s}} d x d y \\
-2 \int_{\Omega} u u_{t} \log |u| d x-\int_{\Omega} u u_{t} d x+\int_{\Omega} u u_{t} d x-\int_{\Omega} u u_{t} d x=-2\left\|u_{t}\right\|_{L^{2}(\Omega)}^{2}-\int_{\Omega} u u_{t} d x \\
=-2\left\|u_{t}\right\|_{L^{2}(\Omega)}^{2}+I(u) \leq I(u) . \quad(4.17)
\end{gathered}
$$

By using Grönwall-Bellman's inequality and $I\left(u_{0}\right)<0$ we have

$$
I(u) \leq I\left(u_{0}\right) e^{t} \leq I\left(u_{0}\right)<0, \quad \forall t \in(0,+\infty) .
$$

It means that $I(u(x, t))$ is decreasing functional with respect to the argument $t$. Let us set

$$
A(t)=\int_{0}^{t}\|u\|_{L^{2}(\Omega)}^{2} d t, A^{\prime}(t)=\|u\|_{L^{2}(\Omega)}^{2}
$$

and by Definition 4.1 we have

$$
A^{\prime \prime}(t)=2 \int_{\Omega} u u_{t} d x=-2[u]_{s}+2 \int_{\Omega} u^{2} \log |u| d x=-2 I(u) .
$$

A simple calculation gives

$$
(\log A(t))^{\prime}=\frac{A^{\prime}(t)}{A(t)}, \quad(\log A(t))^{\prime \prime}=\frac{A^{\prime \prime}(t) A(t)-\left(A^{\prime}(t)\right)^{2}}{A^{2}(t)} .
$$

Now let us estimate $\frac{A^{\prime \prime}(t) A(t)-\left(A^{\prime}(t)\right)^{2}}{A^{2}(t)}$. By using 4.19, 4.6 and Lemma 4.3 , we obtain

$$
A^{\prime \prime}(t)=-2 I(u)=-4 J(u)+A^{\prime}(t)=-4 J\left(u_{0}\right)+4 \int_{0}^{t}\left\|u_{\tau}\right\|_{L^{2}(\Omega)}^{2} d \tau+A^{\prime}(t) .
$$


Similarly, from 4.19 we obtain

$$
\begin{gathered}
\left(A^{\prime}(t)\right)^{2}=\|u\|_{L^{2}(\Omega)}^{4}=\|u\|_{L^{2}(\Omega)}^{4}+2\|u\|_{L^{2}(\Omega)}^{2}\left\|u_{0}\right\|_{L^{2}(\Omega)}^{2}-2\|u\|_{L^{2}(\Omega)}^{2}\left\|u_{0}\right\|_{L^{2}(\Omega)}^{2} \\
+\left\|u_{0}\right\|_{L^{2}(\Omega)}^{4}-\left\|u_{0}\right\|_{L^{2}(\Omega)}^{4}=\left(\int_{\Omega}\left(u^{2}-u_{0}^{2}\right) d x\right)^{2}+2\|u\|_{L^{2}(\Omega)}^{2}\left\|u_{0}\right\|_{L^{2}(\Omega)}^{2}-\left\|u_{0}\right\|_{L^{2}(\Omega)}^{4} \\
=\left(\int_{\Omega} \int_{0}^{t} \frac{\partial u^{2}}{\partial \tau} d \tau d x\right)^{2}+2\|u\|_{L^{2}(\Omega)}^{2}\left\|u_{0}\right\|_{L^{2}(\Omega)}^{2}-\left\|u_{0}\right\|_{L^{2}(\Omega)}^{4}=4\left(\int_{0}^{t} \int_{\Omega} u_{\tau} u d x d \tau\right)^{2} \\
+2\|u\|_{L^{2}(\Omega)}^{2}\left\|u_{0}\right\|_{L^{2}(\Omega)}^{2}-\left\|u_{0}\right\|_{L^{2}(\Omega)}^{4} .
\end{gathered}
$$

Finally, we have

$$
\left(A^{\prime}(t)\right)^{2}=4\left(\int_{0}^{t} \int_{\Omega} u_{\tau} u d x d \tau\right)^{2}+2\|u\|_{L^{2}(\Omega)}^{2}\left\|u_{0}\right\|_{L^{2}(\Omega)}^{2}-\left\|u_{0}\right\|_{L^{2}(\Omega)}^{4} .
$$

It follows that

$$
\begin{aligned}
A^{\prime \prime}(t) A(t) & -\left(A^{\prime}(t)\right)^{2}=-4 J\left(u_{0}\right) A(t)+4 \int_{0}^{t}\left\|u_{\tau}\right\|_{L^{2}(\Omega)}^{2} d \tau A(t)+A^{\prime}(t) A(t) \\
& -4\left(\int_{0}^{t} \int_{\Omega} u_{\tau} u d x d \tau\right)^{2}-2\|u\|_{L^{2}(\Omega)}^{2}\left\|u_{0}\right\|_{L^{2}(\Omega)}^{2}+\left\|u_{0}\right\|_{L^{2}(\Omega)}^{4} \\
& =4\left(\int_{0}^{t}\left\|u_{\tau}\right\|_{L^{2}(\Omega)}^{2} d \tau \int_{0}^{t}\|u\|_{L^{2}(\Omega)}^{2}-\left(\int_{0}^{t} \int_{\Omega} u_{\tau} u d x d \tau\right)^{2}\right) \\
& -4 J\left(u_{0}\right) A(t)+A^{\prime}(t) A(t)-2\left\|u_{0}\right\|_{L^{2}(\Omega)}^{2} A^{\prime}(t)+\left\|u_{0}\right\|_{L^{2}(\Omega)}^{4} .
\end{aligned}
$$

By using the Cauchy-Bunyakovsky-Schwarz inequality, we get

$$
\begin{aligned}
A^{\prime \prime}(t) A(t) & -\left(A^{\prime}(t)\right)^{2}=4\left(\int_{0}^{t}\left\|u_{\tau}\right\|_{L^{2}(\Omega)}^{2} d \tau \int_{0}^{t}\|u\|_{L^{2}(\Omega)}^{2}-\left(\int_{0}^{t} \int_{\Omega} u_{\tau} u d x d \tau\right)^{2}\right) \\
& -4 J\left(u_{0}\right) A(t)+A^{\prime}(t) A(t)-2\left\|u_{0}\right\|_{L^{2}(\Omega)}^{2} A^{\prime}(t)+\left\|u_{0}\right\|_{L^{2}(\Omega)}^{4} \\
& \geq A^{\prime}(t)\left(\frac{A(t)}{2}-\left\|u_{0}\right\|_{L^{2}(\Omega)}^{2}\right)+A(t)\left(\frac{A^{\prime}(t)}{2}-4 J\left(u_{0}\right)\right) .
\end{aligned}
$$

From 4.19, 4.20 and $I(u) \leq I\left(u_{0}\right)<0$, we obtain

$$
\begin{aligned}
& A^{\prime}(t)=A^{\prime}(0)-2 \int_{0}^{t} I(u(x, \tau)) d \tau=-2 I\left(u_{0}\right) t \geq 0, \quad t \geq 0, \\
& A(t)=-I\left(u_{0}\right) t^{2} \geq 0, \quad t \geq 0 .
\end{aligned}
$$


By using 4.27) and (4.6) in 4.26), we calculate

$$
\begin{aligned}
A^{\prime \prime}(t) A(t) & -\left(A^{\prime}(t)\right)^{2} \geq A^{\prime}(t)\left(\frac{A(t)}{2}-\left\|u_{0}\right\|_{L^{2}(\Omega)}^{2}\right)+A(t)\left(\frac{A^{\prime}(t)}{2}-4 J\left(u_{0}\right)\right) \\
& \geq A^{\prime}(t)\left(\frac{-I\left(u_{0}\right) t^{2}}{2}-\left\|u_{0}\right\|_{L^{2}(\Omega)}^{2}\right)+A(t)\left(-I\left(u_{0}\right) t-4 J\left(u_{0}\right)\right) \\
& \geq A^{\prime}(t)\left(\frac{-I\left(u_{0}\right) t^{2}}{2}-\left\|u_{0}\right\|_{L^{2}(\Omega)}^{2}\right)+A(t)\left(-I\left(u_{0}\right) t-2 I\left(u_{0}\right)-\left\|u_{0}\right\|_{L^{2}(\Omega)}^{2}\right) \\
& \geq A^{\prime}(t)\left(\frac{-I\left(u_{0}\right) t^{2}}{2}-\left\|u_{0}\right\|_{L^{2}(\Omega)}^{2}\right)+A(t)\left(-I\left(u_{0}\right)(t+2)-\left\|u_{0}\right\|_{L^{2}(\Omega)}^{2}\right) .
\end{aligned}
$$

From Definition 4.1, we have that $u_{0} \in H_{0}^{s}(\Omega)$ and let

$$
t>t_{0}=\max \left\{\frac{\left\|u_{0}\right\|_{L^{2}(\Omega)}^{2}}{-I\left(u_{0}\right)}-2, \frac{\sqrt{2}\left\|u_{0}\right\|_{L^{2}(\Omega)}}{\sqrt{-I\left(u_{0}\right)}}\right\} \geq 0 .
$$

Let us consider the case

$$
t_{0}=\max \left\{\frac{\left\|u_{0}\right\|_{L^{2}(\Omega)}^{2}}{-I\left(u_{0}\right)}-2, \frac{\sqrt{2}\left\|u_{0}\right\|_{L^{2}(\Omega)}}{\sqrt{-I\left(u_{0}\right)}}\right\}=\frac{\sqrt{2}\left\|u_{0}\right\|_{L^{2}(\Omega)}}{\sqrt{-I\left(u_{0}\right)}} .
$$

By using this fact in 4.28), we obtain

$$
\begin{aligned}
A^{\prime \prime}(t) A(t) & -\left(A^{\prime}(t)\right)^{2} \\
& \geq A^{\prime}(t)\left(\frac{-I\left(u_{0}\right) t^{2}}{2}-\left\|u_{0}\right\|_{L^{2}(\Omega)}^{2}\right)+A(t)\left(-I\left(u_{0}\right)(t+2)-\left\|u_{0}\right\|_{L^{2}(\Omega)}^{2}\right) \\
& \geq A^{\prime}(t)\left(\frac{-I\left(u_{0}\right) t_{0}^{2}}{2}-\left\|u_{0}\right\|_{L^{2}(\Omega)}^{2}\right)+A(t)\left(-I\left(u_{0}\right)\left(t_{0}+2\right)-\left\|u_{0}\right\|_{L^{2}(\Omega)}^{2}\right) \\
& =A^{\prime}(t)\left(\left\|u_{0}\right\|_{L^{2}(\Omega)}^{2}-\left\|u_{0}\right\|_{L^{2}(\Omega)}^{2}\right)+A(t)\left(-I\left(u_{0}\right)\left(t_{0}+2\right)-\left\|u_{0}\right\|_{L^{2}(\Omega)}^{2}\right) \\
& =A(t)\left(-I\left(u_{0}\right)\left(t_{0}+2\right)-\left\|u_{0}\right\|_{L^{2}(\Omega)}^{2}\right) \\
& \geq A(t)\left(-I\left(u_{0}\right)\left(\frac{\left.\left.\left\|u_{0}\right\|_{L^{2}(\Omega)}^{2}-2+2\right)-\left\|u_{0}\right\|_{L^{2}(\Omega)}^{2}\right)}{-I\left(u_{0}\right)}\right)\right. \\
& =0 .
\end{aligned}
$$

Thus, we get

$$
A^{\prime \prime}(t) A(t)-\left(A^{\prime}(t)\right)^{2} \geq 0
$$

Similarly, in the other case

$$
t_{0}=\max \left\{\frac{\left\|u_{0}\right\|_{L^{2}(\Omega)}^{2}}{-I\left(u_{0}\right)}-2, \frac{\left\|u_{0}\right\|_{L^{2}(\Omega)}}{\sqrt{-I\left(u_{0}\right)}}\right\}=\frac{\left\|u_{0}\right\|_{L^{2}(\Omega)}^{2}}{-I\left(u_{0}\right)}-2,
$$

we get

$$
A^{\prime \prime}(t) A(t)-\left(A^{\prime}(t)\right)^{2} \geq 0
$$


So we have

$$
(\log A(t))^{\prime \prime}=\frac{A^{\prime \prime}(t) A(t)-\left(A^{\prime}(t)\right)^{2}}{A^{2}(t)}
$$

and integrating over $\left(t_{0}, t\right)$, we get

$$
(\log A(t))^{\prime}-\left.(\log A(t))^{\prime}\right|_{t=t_{0}}=\int_{t_{0}}^{t} \frac{A^{\prime \prime}(\tau) A(\tau)-\left(A^{\prime}(\tau)\right)^{2}}{A^{2}(\tau)} d \tau \geq 0 .
$$

Thus, we have

Similarly, we get

$$
(\log A(t))^{\prime} \geq\left.(\log A(t))^{\prime}\right|_{t=t_{0}}
$$

$$
\frac{A^{\prime}\left(t_{0}\right)}{A\left(t_{0}\right)}\left(t-t_{0}\right)=\left.(\log A(t))^{\prime}\right|_{t=t_{0}}\left(t-t_{0}\right) \leq \int_{t_{0}}^{t} \log (A(\tau))^{\prime} d \tau=\log (A(t))-\log \left(A\left(t_{0}\right)\right) .
$$

Finally, we arrive at

By summarising above facts 4.37)-4.39 with $t \geq t_{0}$, we compute

$$
\begin{aligned}
& \|u\|_{L^{2}(\Omega)}^{2}=A^{\prime}(t)=(\log A(t))^{\prime} A(t) \geq\left.(\log A(t))^{\prime}\right|_{t=t_{0}} A(t)=\frac{A^{\prime}\left(t_{0}\right)}{A\left(t_{0}\right)} A(t)=\frac{A(t)}{A\left(t_{0}\right)} A^{\prime}\left(t_{0}\right) \\
& \geq A^{\prime}\left(t_{0}\right) e^{\frac{A^{\prime}\left(t_{0}\right)}{A\left(t_{0}\right)}\left(t-t_{0}\right)}=\left\|u\left(\cdot, t_{0}\right)\right\|_{L^{2}(\Omega)}^{2} e^{\frac{A^{\prime}\left(t_{0}\right)}{A\left(t_{0}\right)}\left(t-t_{0}\right)} \geq\left\|u_{0}\right\|_{L^{2}(\Omega)}^{2} e^{\frac{A^{\prime}\left(t_{0}\right)}{A\left(t_{0}\right)}\left(t-t_{0}\right)} .
\end{aligned}
$$

That is,

$$
\lim _{t \rightarrow+\infty}\|u(t, \cdot)\|_{L^{2}(\Omega)}^{2}=+\infty
$$

Remark 4.5. In the Abelian (Euclidean) case $\mathbb{G}=\left(\mathbb{R}^{N},+\right)$, we have $Q=N$ and $|\cdot|=|\cdot|_{E}\left(|\cdot|_{E}\right.$ is the Euclidean distance), if $s \rightarrow 1^{-}$we get blow-up result at infinity in [3] and [10].

\section{REFERENCES}

[1] B. Abdellaoui and R. Bentifour. Caffarelli-Kohn-Nirenberg type inequalities of fractional order with applications. J. Funct. Anal., 272(10):3998-4029, 2017.

[2] L. A. Caffarelli, R. Kohn and L. Nirenberg. First order interpolation inequalities with weights. Composito Math., 53(3):259-275, 1984.

[3] H. Chen, P. Luo and G. Liu. Global solution and blow-up of a semilinear heat equation with logarithmic nonlinearity. J. Math. Anal. Appl., 422:84-98, 2015.

[4] H. Chen and S. Tian. Initial boundary value problem for a class of semilinear pseudo-parabolic equaitons with logarithmic nonlinearity. J. Differential Equations, 258:4424-4442, 2015.

[5] J. Chen and E. M. Rocha. A class of sub-elliptic equations on the Heisenberg group and related interpolation inequalities. Oper. Theory Adv. Appl., 229:123-137, Birkhäuser/Springer Basel AG, Basel, 2013.

[6] E. Di Nezza, G. Palatucci and E. E. Valdinoci. Hitchhiker's guide to the fractional Sobolev spaces. Bulletin des Sciences Mathematiques, 136(5):521-573, 2012.

[7] G. B. Folland and E. M. Stein. Hardy spaces on homogeneous groups. Mathematical Notes, Vol. 28, Princeton University Press, Princeton, N.J.; University of Tokyo Press, Tokyo, 1982.

[8] E. Gagliardo. Ulteriori proprietà di alcune classi di funzioni in più variabili. Ricerche Mat., 8:24-51, 1959 . 
[9] H. Hajaiej, X. Yu and Z. Zhai. Fractional Gagliardo-Nirenberg and Hardy inequalities under Lorentz norms. J. Math. Anal. Appl., 396(2), 569-577, 2012.

[10] Y. Han. Blow-up at infinity of solutions to a semilinear heat equation with logarithmic nonlinearity. J. Math. Anal. Appl., 474(1):513-517, 2019.

[11] C. Ji, J. Yin and Y.Cao. Instability of positive periodic solutions for semilinear pseudoparabolic equations with logarithmic nonlinearity. J. Differential Equations, 26(1):5446-5464, 2016.

[12] A. Kassymov and D. Suragan. Some functional inequalities for the fractional p-sub-Laplacian. arXiv:1804.01415v2, 2018.

[13] A. Kassymov, M. Ruzhansky and D. Suragan. Hardy-Littlewood-Sobolev and Stein-Weiss inequalities on homogeneous Lie groups. Integral Transforms and Special Functions, 30(8):643655, 2019.

[14] A. Kassymov, M. Ruzhansky and D. Suragan. Anisotropic fractional Gagliardo-Nirenberg, weighted Caffarelli-Kohn-Nirenberg and Lyapunov-type inequalities, and applications to Riesz potentials and $p$-sub-Laplacian systems. arXiv:1806.08940, 2018.

[15] J. Merker. Generalizations of logarithmic Sobolev inequalities. Discrete Contin. Dyn. Syst. Ser. S, 1(2): 329-338, 2008.

[16] L. Nirenberg. On elliptic partial differential equations. Ann. Scuola Norm. Sup. Pisa (3), $13: 115-162,1959$.

[17] H.-M. Nguyen, M. Squassina. Fractional Caffarelli-Kohn-Nirenberg inequalities. J. Funct. Anal., 274:2661-2672, 2018.

[18] M. Ruzhansky and D. Suragan. Hardy and Rellich inequalities, identities, and sharp remainders on homogeneous groups. Adv. Math., 317, 799-822, 2017.

[19] M. Ruzhansky, D. Suragan and N. Yessirkegenov. Extended Caffarelli-Kohn-Nirenberg inequalities, and remainders, stability, and superweights for $L^{p}$-weighted Hardy inequalities. Trans. Amer. Math. Soc. Ser. B, 5:32-62, 2018.

[20] M. Ruzhansky, N. Tokmagambetov, N. Yessirkegenov, Best constants in Sobolev and Gagliardo-Nirenberg inequalities on graded groups and ground states for higher order nonlinear subelliptic equations. arXiv:1704.01490v1, 2018.

[21] M. Ruzhansky, D. Suragan and N. Yessirkegenov. Extended Caffarelli-Kohn-Nirenberg inequalities and superweights for $L^{p}$-weighted Hardy inequalities. C. R. Math. Acad. Sci. Paris, 355(6):694-698, 2017.

[22] M. Ruzhansky, D. Suragan and N. Yessirkegenov. Caffarelli-Kohn-Nirenberg and Sobolev type inequalities on stratified Lie groups. NoDEA Nonlinear Differential Equations Appl., 24(5):Art. $56,2017$.

[23] M. Ruzhansky and D. Suragan. On horizontal Hardy, Rellich, Caffarelli-Kohn-Nirenberg and p-sub-Laplacian inequalities on stratified groups. J. Differential Equations, 262:1799-1821, 2017.

[24] Ruzhansky M., Suragan D.: Hardy inequalities on homogeneous groups. Progress in Math. Vol. 327, Birkhäuser, 588 pp, (2019) (open access book)

[25] T. Feng, P. Niu and J. Qiao. Several logarithmic Caffarelli-Kohn-Nirenberg inequalities and applications. J. Math. Anal. Appl., 457:822-840, 2018.

[26] S. Zhang, Y. Han and J. Dou. A class of Caffarelli-Kohn-Nirenberg type inequalities on the H-type group. Sem. Mat. Univ. Padova, 132:249-266, 2014.

Aidyn Kassymov:

Al-Farabi Kazakh National University

71 Al-Farabi aVEnUe

050040 Almaty

KAZAKHSTAN

AND

Department of Mathematics: Analysis, Logic and Discrete Mathematics

Ghent University, Belgium

AND 
Institute of Mathematics and Mathematical Modeling

125 PUSHKIN STR.

050010 Almaty

KAZAKHSTAN

E-mail address kassymov@math.kz

Michael RuZhansky:

Department of Mathematics: Analysis, Logic and Discrete Mathematics Ghent University, Belgium

AND

School of Mathematical Sciences

QUEEN MARY UNIVERSITY OF LONDON

United KingDom E-mail address michael.ruzhansky@ugent.be

DurvudKhan Suragan:

DePaRtMENT OF MATHEMATiCs

NAZARBAYEV UNIVERSITY

53 Kabanbay Batyr Ave, Nur-Sultan 010000

KAZAKHSTAN

E-mail address durvudkhan.suragan@nu.edu.kz 